Cite as: Wallace, J.R. \& Scott, S.D. (2008). Contextual Design Considerations for Co-located, Collaborative Tables. In Proceedings of IEEE Workshop on Tabletop and Interactive Surfaces 2008, October 1-3, 2008, Amsterdam, The Netherlands.

\title{
Contextual Design Considerations for Co-located, Collaborative Tables
}

\author{
James R. Wallace and Stacey D. Scott \\ Department of Systems Design Engineering \\ University of Waterloo, ON, Canada \\ \{jrwallac,s9scott\}@uwaterloo.ca
}

\begin{abstract}
To date, digital tabletop research has predominantly focused on resolving fundamental software and hardware challenges introduced by this new interactive platform. Understanding not only what technical functionality a digital tabletop can provide, but also how appropriate that functionality is for different usage contexts is crucial in designing tables intended for use outside of the research lab. In this paper, we propose five contextual factors to consider in the tabletop design process - social and cultural, activity, temporal, ecological, and motivational - and discuss how these factors influence the design of three main aspects of tabletop systems: software interface, physical form, and connectedness. This work provides a means for tabletop designers to understand the factors that impact the applicability of existing and future design approaches for a given context of use.
\end{abstract}

\section{Introduction}

As digital tabletop systems mature and move beyond the research laboratory, important challenges remain to secure user adoption. To date, the community has mainly focused on technical challenges in realizing robust tabletop systems, including multi-user and multi-touch input $[6,8,15,50]$, new display technologies [23], object recognition and tracking [33, 35] and resolving software interface challenges introduced by a large, horizontal workspace, such as orientation [16, 44] and reach [31, 44]. These efforts have led to a wide variety of tabletop platforms, software interface tools and interaction techniques. In order to begin combining these technologies into useful, real-world tabletop applications, it is critical to consider the appropriateness of each tool or design approach in its context of use.

This paper is motivated by the current lack of advice in the literature addressing 1) how to apply existing (and future) tabletop hardware and software technologies to different usage contexts in order to achieve appropriate form and function, and 2) the possible design variations that will likely be necessary across these different contexts. To address these issues, we reflect on five contextual factors relevant to the tabletop design process: social and cultural, activity, temporal, environmental, and motivational. These contextual factors are derived from the basic who, what, when, where, and why questions that are commonly used to understand the user context and establish design requirements during a user-centered design process [42].

To demonstrate how contextual factors can influence the appropriateness of existing tabletop hardware and software technologies, we discuss critical design issues within each factor and explain how they impact three fundamental aspects of tabletop design: the software interface, the physical form, and the connectedness of the table to its surrounding context.

\section{Contextual Design Considerations}

Establishing design requirements tailored to an interactive system's intended usage context is important for producing effective, usable technology and for fostering user adoption [42]. To help understand how context impacts the design of interactive tabletop systems, we reflect on five usage contexts for designers to consider during the requirements establishment process. For each context, we identify specific design considerations and examine their impact on the table's software interface, its physical form, and its connectedness to other devices.

With respect to software interfaces, we examine how these contextual factors influence the appropriate complexity of a table's visual interface and the interactions required by the user. This complexity is in part determined by the experience users can be expected to have with the interface or interaction style. Previous experiences with conventional tables can also be leveraged to provide simpler, more intuitive interfaces. Hilleges et al. [18] call this designing for pseudo-physicality, or making objects behave like their physical counterparts. For example, the Rotate and Translate (RNT) and two-finger rotation techniques [16] enable digital object rotation using one or two finger manipulation similar to techniques used on physical objects. Use of tangible user interfaces (TUIs), which enable users to interact with physical objects to affect the digital workspace, can also 
Cite as: Wallace, J.R. \& Scott, S.D. (2008). Contextual Design Considerations for Co-located, Collaborative Tables. In Proceedings of IEEE Workshop on Tabletop and Interactive Surfaces 2008, October 1-3, 2008, Amsterdam, The Netherlands.

simplify interaction by leveraging real world experiences [35]. More complex, computationally powerful interfaces are also possible with metaphysical design approaches that leverage digital capabilities to provide enhanced functionality [18]. For example, computationally enforcing rules in a tabletop game to constrain user behaviour [37].

With respect to the table's physical form, we examine how the contextual factors influence the design requirements related to a table's width, height, length, shape, display angle, and aesthetic design. While these aspects of design are the primary considerations in non-digital tables, they are often secondary considerations for interactive surfaces. Yet, longitudinal and in-field research has found that the table's physical form impacts users' impressions of the table's viability, and that more familiar form factors are appropriate for use in for everyday life [27, 51]. Clearly, form will play a crucial role in securing widespread user adoption of this new interaction platform.

With respect to the table's connectedness, we examine how the contextual factors influence the degree to which the table needs to be aware of or be able to interface with other objects, surfaces, or devices in the usage context. This issue has, so far, received little attention in the tabletop literature, with some notable exceptions [3, 30, 32, 52]. Yet, tabletop connectedness has previously been identified as an important design consideration for collaborative, colocated tabletop systems as a means to support transitions between tabletop activities and activities performed elsewhere, and as a means to support the use of other digital devices or non-digital objects that users may bring to the table [41].

\subsection{Social \& Cultural Context}

The social and cultural context refers to the factors that impact the social and cultural norms that govern group behaviour in a given context. For example, the relationship between group members (e.g. professional or personal, peer or supervisor/subordinate), the organizational culture (e.g., casual/home, business, military), and the national or regional culture (e.g., North America vs. Japan, or inner city vs. rural), can all influence the expected behavioural norms within a group. The size of a group can also influence group behaviour and social norms [38].

Each of these considerations (and combinations thereof) has implications for a table's interface, form, and connectedness. For example, the relationship, organizational culture, and national/regional culture impact how formal a social situation is. This in turn impacts how serious the social or professional consequences may be if a group member is seen struggling with a complex tabletop interface. An oft touted advantage of tables can be problematic for some social and cultural contexts: their large, shared surface, which promotes group awareness by providing highly visible actions, also exposes one's mistakes to others [20], and in some contexts, can invite criticism of one's actions and data [53]. Further impacts of the social and cultural context on table design are discussed below.

\subsubsection{Software Interface}

To minimize the potential for negative social or professional consequences, tables situated in formal social or cultural contexts should provide intuitive, easy to use interfaces that minimize opportunities for mistakes or uncertainty. Even in usage contexts where users are likely to have a great deal of training and experience with the technology, intense social pressure can fluster even the most competent user [55], and thus should be anticipated during the design process. Interfaces that provide simple interface components with obvious visual affordances, and simple interaction that draws on well ingrained interaction practices, such as the pseudo-physical design approach mentioned above, would help to address this design concern.

The recent trend toward multi-finger or bimodal interaction [15, 49] may be problematic in formal social and cultural contexts, as a recent study suggests that complex gestures beyond single-touch pointing and dragging are not consistently performed across users, and thus are not robustly recognized [8].

In less formal situations where the consequences of making mistakes may be less severe, providing a means for "socially safe" interactions may still be desirable. Tang [47] observed that people use "personal" spaces (or territories [39]) on conventional tables to explore ideas they are not yet ready to share with other group members. Providing similar support for territory formation and personal interactions with task content, along with mechanisms to integrate this content, when desired, into the shared group activity, may help to enable socially safe interaction. Providing pseudo-physical interaction with tabletop objects, as well as localized functionality, such as using in-context menus $[19,25,40]$, or easy resizing of workspace content, may also enable this behaviour.

The table's interface should also account for the personal and professional relationships between group members in a given context. For example, parent/child or teacher/student relationships may introduce requirements for different user privileges or computerenforced roles [37], or for concentrating some system functionality in a certain tabletop location. Such teaching relationships may also benefit from the inherently high visibility provided on table interfaces, 
Cite as: Wallace, J.R. \& Scott, S.D. (2008). Contextual Design Considerations for Co-located, Collaborative Tables. In Proceedings of IEEE Workshop on Tabletop and Interactive Surfaces 2008, October 1-3, 2008, Amsterdam, The Netherlands.

as open interactions can facilitate learning through observation [21].

\subsubsection{Form}

The table's form must also account for social and cultural contextual considerations. For example, aesthetic design may be crucial for settings in which representatives of an organization are trying to impress a group of visitors or for settings where groups of friends come to relax and have fun together. The size, shape, and height of the table's physical form are also influenced by social and cultural factors. For example, in certain cultures, or in preschool contexts, tables are commonly situated low to the floor.

Appropriate table size is also influenced by cultural and age considerations: the distance at which people are comfortable interacting with others varies across age and culture. For example, Nordic people prefer to interact at further interpersonal distances than those from Latin regions [14], and would likely feel more socially comfortable, especially in formal situations or when interacting with strangers, at larger tables. In contrast, children enjoy close interaction with others; thus smaller tabletops would be appropriate for elementary schools, such as SMART Technologies' early learner table (its aesthetic design resembles a large Fisher Price ${ }^{\circledR}$ toy) [48]. The table must also be sufficiently large to comfortably accommodate common group sizes in the given context.

\subsubsection{Connectedness}

In formal social and cultural contexts, where exposure of one's mistakes or even "personal" interactions may result in criticism or have other negative consequences, it may be appropriate to provide tabletop connections to nearby personal devices (e.g. laptop, handheld computer) [43]. This capability enables "safe", private interactions until one is ready to expose the results. For example, a member of a large group could use a laptop to fumble through their file system to find a document they wish to share without exposing these interactions, or any of their personal data that may also be stored there. Though this design approach is likely to reduce awareness, it may be a reasonable tradeoff where the potential for social or professional consequences are high.

\subsection{Activity Context}

The activity context refers to the type of task or activity in which the group is engaged, and the characteristics of that activity that influence tabletop design. Not surprisingly, the activity context has significant influence on the table's design, since it is the main driver of the table's functional requirements. Consequently, the tabletop community has primarily focused on this context in order to enable core software and hardware interactions with digital tabletop objects potentially common to many tabletop activities. This section builds on this work by discussing contextspecific considerations that impact the table's functional and non-functional requirements.

Many aspects of the activity context influence these requirements, including whether an activity is open or closed [36], requires tight or loosely coupled actions [46], is cooperative or competitive, or requires access to local or remote data, media, or tools (digital or non-digital). The impact of these considerations on the table's design is discussed below.

\subsubsection{Software Interface}

In addition to basic functional requirements derived from traditional task analyses, other characteristics of the activity context, such as those discussed above, have significant impact on the appropriate design approach used to address those basic functional requirements in the software interface.

The openness, or the degree of structure, of an activity, impacts the appropriate level of interface complexity. An open task, such as medical decisionmaking may require access to a variety of data sources and involve a wide range of personnel who may or may not be familiar with the technology. Thus, interfaces for such contexts should provide intuitive interfaces and obvious means to access alternative data sources (since the required type of data and source may vary from one patient case to another).

In contrast, closed activities, such as ship navigation or air traffic control, involve well structured, often recurring, problems and typically involve a predetermined set of tools [36]. These activities involve specialized personnel who typically receive significant training; thus, more complex interfaces and interactions may be appropriate.

The level of task coupling required in an activity also impacts interface design. Tightly coupled activities, where group members' actions are highly interdependent, are likely to require interfaces that provide high levels of workspace awareness [12]. Visible group interactions facilitate workspace awareness [39], which may be addressed by providing direct, modeless interaction, such as pseudo-physical techniques, as this design approach helps to promote awareness and prediction of group member's actions [13], and prevent surprises in a shared workspace [7].

\subsubsection{Physical Form}

The set of activities that a table must support also impacts its physical form requirements. For example, the level of required coupling within a given group activity can impact the table's size requirements. 
Cite as: Wallace, J.R. \& Scott, S.D. (2008). Contextual Design Considerations for Co-located, Collaborative Tables. In Proceedings of IEEE Workshop on Tabletop and Interactive Surfaces 2008, October 1-3, 2008, Amsterdam, The Netherlands.

Loosely coupled tabletop activities, such as a meeting where group members are taking individual notes, will likely require less workspace awareness (or at least less continual awareness); thus, providing a larger table with sufficient room for each member to work individually, or providing strong connections to separate, personal devices, may be appropriate.

The table's form must also account for the size and amount of materials (e.g., data, media, and tools) needed to perform the activity. For example, activities that involve analysis of map data or comparison of large visual data sets will require a sufficiently large table to enable the group to overview the data [5].

The required level of cooperation in an activity can also impact the table's form. In particular, it impacts the people's preferred seating arrangement during the activity. During cooperative activities, people prefer to sit either side-by-side or at adjacent sides of the table, while they prefer to sit on opposite sides of a table during competitive activities [41, 45]. The table's form should support these preferences for a given activity context, for example, through easy access to appropriate sides that are free from encumbrances such as projection equipment, or alternative display attachments.

Competitive activities may also introduce requirements for displaying private information. For special purpose (e.g. gaming) tables, incorporating small, angled personal displays along different sides of the table, or using directed display techniques to provide position-dependent, tailored views [26], may be appropriate.

\subsubsection{Connectedness}

The table's required connectedness is heavily influenced by the activity context. In particular, the type and source of materials needed (or desired) to accomplish the activity will drive the required level of support the table must provide for local device discovery, for accessing local or networked data sources (or the Internet in general), for recognizing and/or tracking (or ignoring) non-digital objects, and for connecting to nearby displays.

How often a group will need access to or make use of any given external materials will guide the permanence of, or level of ease to establish, the connectivity between the table and the external materials. For example, tables used to share personal media, such as photo-sharing activities, should provide simple, plug-and-play support for common device connections, such as USB connections [22].

The degree of openness of an activity also impacts the connectedness requirements. Open activities are more likely to involve a wider, less predictable range of materials and users; thus broad support should be provided for connectivity to various, common local and networked devices, as well as general Internet support for accessing alternative, web-accessible sources.

\subsection{Temporal Context}

The temporal context refers to how often and how long groups are likely to use the table, as well as how much time pressure groups are under when performing tabletop activities. For example, are typical users likely to use the table once or rarely, or are they likely to use it daily? Will they use the table for short (e.g. seconds to a few minutes) tabletop activities, or will they use the table for long periods of time? These temporal considerations impact the amount of experience one can assume users will have with the system, which, as discussed earlier, impacts the level of interface and interaction complexity that will be appropriate for the table design. They also have significant impact on the table's ergonomic requirements.

Another important temporal consideration is whether a group is under significant time pressure to complete their tabletop activities. This consideration impacts the level of stress the group is under, which in turn can impact their cognitive functioning [54]. The impacts of these temporal considerations on the table's design are discussed below.

\subsubsection{Software Interface}

In brief or infrequent usage situations, where lack of prior experience or limited time for recalling system functionality is likely, interface designs that offer intuitive interfaces with minimal requirements for "at the table" learning are desirable. Providing limited, visually obvious functionality, or drawing from previous experiences with conventional tables (e.g. with pseudo-physical designs), would help address this design concern.

In usage contexts where brief, but frequent, table activities are expected, providing interfaces and interaction that support progression towards more efficient interaction as users gain more experience, may be appropriate. For example, Flow Menus [11], developed for interaction with wall displays, provide in-context menus that a naïve user can use through gesture selection of visual menu items, and an experienced user can use by rapidly gesturing the shape that corresponds to a set of desired menu selections. With this approach, an experienced user can always fall back on the simpler method of menu selection after time away from the table or when in a degraded cognitive state. 
Cite as: Wallace, J.R. \& Scott, S.D. (2008). Contextual Design Considerations for Co-located, Collaborative Tables. In Proceedings of IEEE Workshop on Tabletop and Interactive Surfaces 2008, October 1-3, 2008, Amsterdam, The Netherlands.

This design approach may also be appropriate for contexts where prolonged use is expected; as users become more proficient over time, they are likely to want more efficient interactions. Prolonged table usage also provides opportunities for users to discover new features and learn complex interaction techniques; thus, a more computationally powerful and visually sophisticated interface may be appropriate. For example, complex gestures that accommodate metaphysical interaction, such as enabling item copying through a two finger gesture [1], may be appropriate when prolonged or frequent table usage is expected.

The time constraints of a given context also impact the appropriate level of interface complexity. Under few time restrictions, groups are free to explore the interface at their leisure; thus more complex interfaces and interaction techniques, as discussed above, may be appropriate. In contrast, activities performed under significant time pressure are likely to provide few opportunities for groups to explore or learn the system's capabilities. Interfaces that provide minimalistic visual designs that reduce visual clutter and that emphasize key functionality and data interaction capabilities are desirable for these contexts.

\subsubsection{Form}

The table's form is also influenced by the temporal considerations discussed above. If prolonged or frequent table use is likely, a high priority should be placed on providing a physically comfortable table form. For example, prolonged interaction is likely to require a table height, edge design, and input capture mechanism conducive to groups interacting from seated positions around the table.

In contrast, ergonomic concerns are less likely to be a design priority for tables used predominantly for short or infrequent interactions; however, form some aspects of form should still be considered. For example, providing a table height that enables users to stand may be appropriate when brief table interaction is expected in order to facilitate "walk up" use, such as at information kiosks [28].

As the duration and frequency of use increase, so does the likelihood that devices will need to accommodate aspects of everyday life. In a study of long-term, primarily single-user table usage, Wigdor et al. [51] found that a table was tilted to facilitate comfort, and that the angle was dictated not only by ergonomics, but also by how well it could accommodate commonly used physical objects (the angle was not steep enough to let a coffee cup slip off). Similar display angle titling, primarily for ergonomic reasons, was observed in a study of single-user reading activities on a table [29].

\subsubsection{Connectedness}

Temporal considerations also impact the table's data access requirements. Groups using tables for sustained and frequent use are more likely to require access to a wide range of data sources. Along with supporting prolonged and frequent use, massively connected tables that provide robust predetermined device and network connections, as well as ad hoc device discovery, are more likely to be able to cope with unforeseen group needs. Such exception handling is a critical aspect of developing effective groupware that foster user adoption [10].

On the other hand, systems which focus on short, infrequent use are more likely to serve a more specialized purpose. In these cases, data access is likely restricted to an easily anticipated set of data, possibly physically available to users. For example, external data on a mobile device can be accessed via a physical connection [32-34] or external data can be accessed via a network connection [3].

\subsection{Ecological Context}

The ecological context refers to the environment in which the table is situated, the table's role in this environment, and its relationship to other objects and devices in it. For example, is the table the only computational device in the setting, or will groups use other devices (e.g. laptops, cameras, wall displays) in conjunction with the table? Will non-digital artifacts be brought to the table? Is the table located in a family home, in an office building, or on the bridge of a naval ship? Similar to the social and cultural context, these ecological considerations can impact the formality of the situation, and also establish the table's decorative function. They also have significant implications for the table's connectedness to other devices in the environment. The impact of these considerations on the table's design is discussed below.

\subsubsection{Software Interface}

Tables are likely to exist in environments where nondigital objects are present, especially in casual, less structured environments, such as the home. When physical objects are present, tables are unlikely to be used exclusively as digital tables, and instead may be used to temporarily support physical objects, such as coffee cups or loose paper. In these cases, the table's software interface will need to distinguish between intentional and unintentional interaction. Techniques for adapting tabletop interfaces in the presence of physical objects fall under two general categories; those that track objects and automatically adjust the software interface, and those which allow the user to manually adapt the interface. 
Cite as: Wallace, J.R. \& Scott, S.D. (2008). Contextual Design Considerations for Co-located, Collaborative Tables. In Proceedings of IEEE Workshop on Tabletop and Interactive Surfaces 2008, October 1-3, 2008, Amsterdam, The Netherlands.

Automatic interface adapting techniques, such as Display Bubbles [4], may be most appropriate for casual settings since they reduce the amount of effort required by users in managing the interface. In casual settings there are also less severe consequences of the system mistakenly interpreting users' intentions as they interact with physical objects on the table. Automatic techniques reduce the amount of attention users dedicate to managing physical objects, and instead let them focus on their activity and social goals.

Manual techniques such as user drawn path menus [25] are likely to be more appropriate in formal settings or where less predictable interaction with physical objects is likely. Formal scenarios are likely to be less tolerant of system recognition or interface adaption errors, and are settings in which users are more likely to need control over where digital artifacts are relocated. In cases where few physical objects are present, it may be more desirable to simply move the physical object rather than alter the table's interface. Manual display adaptation also enables users to decide when they want the table to simply ignore physical objects located on the table. This capability enables users to make direct visual comparisons between data located on the table interface and data located on a physical device.

\subsubsection{Form}

When purchasing a coffee table, its owners may consider the look and feel of the table to be as important as its functionality - does it match the couch or the end tables? Does the table feel sturdy? Is there enough leg room under it to be able to lay back and watch TV? In casual environments, a table is unlikely to be purchased for its own sake; it will likely replace an existing piece of furniture, and in so doing, must be able to accommodate these concerns as well. In environments where the table's non-digital design requirements are as heavily weighted as its digital design requirements, form is an important design consideration.

In work environments, function will likely be a more important consideration than form. In these cases the table is primarily designed to serve a functional purpose, and decorative function is a secondary concern. When function more closely dictates form, the table is less likely to rely on co-present devices for design cues. A functional table is more likely to have its size dictated by system requirements (screen size, viewing angle, interaction style), than by the external requirements used in the casual case.

\subsubsection{Connectedness}

Connectivity with external devices allows for the transfer of applications and data, and facilitates cross- device interaction; however implementing an environment in which such connectivity is facilitated is a complex matter. In formal settings, an analysis of the devices, users and processes involved may be used to derive connectivity requirements. Understanding the workflow in a collaborative environment can help determine which aspects of the table are most likely to be in demand. For example, if required data are predominantly found on mobile devices, the table may be used largely as an ephemeral display, in which case the primary design concern should be facilitating the use of the table to visualize personal data.

However in casual, less structured environments such an analysis may be impossible. In these settings, standard methods of connectivity must be developed to facilitate inter-device communication. Standards such as Zero Configuration Networking [2] that allow devices to automatically discover and advertise services on the network are useful in this endeavor. These standards allow a heterogeneous collection of devices to seamlessly interact without the use of a complex infrastructure, and facilitate the navigation of network resources in an efficient manner. By utilizing these standards, the technical issues related to networking are removed, leaving the contextual design considerations as the predominant factors in design.

\subsection{Motivational Context}

The motivational context refers to the personal and professional goals that motivate the activity(ies) for which the table is used, including the group's motivation for using the table rather than alterative computing devices. For example, are users motivated by the desire for a fun and socially enjoyable user experience, or are users motivated by the desire to achieve efficient decision making with complex data? These considerations impact the appropriateness of a simple or complex interface and of an aesthetic or functional form. The table's connectedness requirements will stem primarily from the four contexts discussed above, and thus will not be discussed further here.

\subsubsection{Software Interface}

A user's motivation can play a role in their proficiency and user experience when using the table. Tables with complex interfaces risk intimidating, and consequently eliminating, potential users; this consequence is particularly likely in casual settings where users may only choose to use the table based on their own personal motivations. Users who are uninterested in learning the intricacies of a complex system may be discouraged from using the system at all [24]. Thus; 
Cite as: Wallace, J.R. \& Scott, S.D. (2008). Contextual Design Considerations for Co-located, Collaborative Tables. In Proceedings of IEEE Workshop on Tabletop and Interactive Surfaces 2008, October 1-3, 2008, Amsterdam, The Netherlands.

intuitive interfaces and interaction will be critical for obtaining widespread consumer use.

In formal settings, where users may be motivated to use a table based on monetary compensation or their professional reputation, they may be more willing to learn to use a complex interface. However, as discussed above, in certain social and cultural contexts, a complex system can also diminish a users' reputation if they falter while using it in front of others.

In contexts where users are seeking a fun and social experience, providing a more playful look and feel to the table's interface and interaction may be desirable [27].

\subsubsection{Physical Form}

Tables, in the contexts studied here, serve the primary purpose of providing a space for collaboration [17]. Understanding how the physical design of that collaborative space contributes to its understood meaning can help elicit requirements for better design. In formal environments, a users' motivation in using a table highly correlates to its functional purpose - users move to an architectural planning table to work with blueprints and other planning tools. In designing for well structured settings, requirements are generally derived directly from this functional purpose rather than external motivations.

In casual circumstances, the purpose of the table may be motivated by a number of non-functional factors. The table may not be the focus of collaboration - it may simply be a place to order and hold drinks in a bar, or it may be a place for children to congregate and play. In these cases the form of the table is not primarily communicating function, but purpose, atmosphere and style. For example, in any restaurant the function of tables are nearly identical - patrons sit, are served food, converse with their peers, and leave. However the form of the table can influence the atmosphere of the restaurant - ranging from a romantic date to a late night stop at McDonalds with friends.

\section{Discussion and Conclusions}

When considering the appropriateness of including any existing design approach into the design of an interactive system, the answer is always "it depends." This paper attempts to clarify the factors upon which such decisions depend for the design of collaborative, co-located tabletop systems. In particular, we examine five contextual factors - derived from the who, what, when, where, and why of table use - to help identify key tabletop design considerations.

Within each of the contexts considered, we illustrate that a table's interface, form, and connectedness are heavily dependent on the context in which it is being used. The appropriate intuitiveness of an interface depends not only on the need to support group awareness, but also the motivation of the users, the social and cultural context of use and how much experience end users can be expected to have to the system. Similarly, the appropriate physical form of the table may depend on the casualness of those involved, ergonomic and decorative considerations. Finally, the appropriate connectedness of a table may rely on what devices are available in the target environment, privacy concerns, or the ability to fluidly support transitions between those devices and the table.

These considerations illustrate that an ecologically and contextually valid approach to tabletop research can provide critical design information beyond technical limitations. They exemplify the need to "get the right design before getting the design right" [9], and that the right design is highly dependent on factors external to the table itself.

\section{References}

1. Apted, T., J. Kay, \& A. Quigley, Tabletop sharing of digital photographs for the elderly. In CHI 2006, 781-790

2. Cheshire, S. Zero Configuration Networking (Zeroconf). 2008. Available from: www.zeroconf.org (June 2008).

3. Collins, A., T. Apted, \& J. Kay, Tabletop File System Access: Associative and Hierarchical Approaches. In Tabletop 2007, 113-120.

4. Cotting, D. \& M. Gross, Interactive environment-aware display bubbles. In UIST 2006, 245-254.

5. Czerwinski, M., et al., Toward Characterizing the Productivity Benefits of Very Large Displays.. In INTERACT 2003, 9-16.

6. Dietz, P. \& D. Leigh, DiamondTouch: a multi-user touch technology. In UIST 2001, 219-226.

7. Eden, H., E. Scharff, \& E. Hornecker, Multilevel design and role play: experiences in assessing support for neighborhood participation in design. In DIS 2002, 387392.

8. Epps, J., S. Lichman, \& M. Wu, A study of hand shape use in tabletop gesture interaction. In CHI Extended Abstracts 2006, 748-753.

9. Greenberg, S. \& B. Buxton, Usability evaluation considered harmful (some of the time). In CHI 2008, 111-120.

10. Grudin, J., Groupware and social dynamics: eight challenges for developers. Commun. ACM, 1994. 37(1), 92-105.

11. Guimbretiere, F. \& T. Winograd, FlowMenu: combining command, text, and data entry. In UIST 2000, 213-216.

12. Gutwin, C. \& S. Greenberg, Design for individuals, design for groups: tradeoffs between power and workspace awareness. In CSCW 1998, 207-216.

13. Ha, V., et al., Direct Intentions: The Effects of Input Devices on Collaboration around a Tabletop Display. In Tabletop 2006, 177-184.

14. Hall, E.T., The Hidden Dimension. 1966: Anchor Books. 
Cite as: Wallace, J.R. \& Scott, S.D. (2008). Contextual Design Considerations for Co-located, Collaborative Tables. In Proceedings of IEEE Workshop on Tabletop and Interactive Surfaces 2008, October 1-3, 2008, Amsterdam, The Netherlands.

15. Hancock, M., S. Carpendale, \& A. Cockburn, Shallowdepth 3d interaction: design and evaluation of one-, twoand three-touch techniques. In CHI 2007, 1147-1156.

16. Hancock, M.S., et al., Rotation and Translation Mechanisms for Tabletop Interaction. In Tabletop 2006, 79-88.

17. Harrison, S. \& P. Dourish, Re-place-ing space: the roles of place and space in collaborative systems. In $C S C W$ 1996, 67-76.

18. Hilliges, O., et al., Designing for collaborative creative problem solving. In Creativity \& Cognition 2007, 137146.

19. Hinrichs, U., S. Carpendale, \& S.D. Scott, Interface currents: supporting fluent face-to-face collaboration. In SIGGRAPH Sketches 2005.

20. Huang, E.M., E.D. Mynatt, \& J.P. Trimble, When design just isn't enough: the unanticipated challenges of the real world for large collaborative displays. Personal Ubiquitous Computing, 2007, 11(7), 537-547.

21. Hutchins, E., The technology of team navigation. In J. Galegher, R.E. Kraut, \& C. Egido, (eds.). Intellectual Teamwork: Social and technological foundations of cooperative work, 1990, Lawrence Erlbaum Associates, 191-220.

22. Izadi, S., et al., Dynamo: a public interactive surface supporting the cooperative sharing and exchange of media. In UIST 2003, 159-168.

23. Kakehi, Y., T. Naemura, \& M. Matsushita, Tablescape Plus: Interactive Small-sized Vertical Displays on a Horizontal Tabletop Display. In Tabletop 2007, 155-162.

24. Kay, A.C., Computers, Networks and Education. Scientific American, 1991, 265(3), 138-148.

25. Leithinger, D. \& M. Haller, Improving Menu Interaction for Cluttered Tabletop Setups with User-Drawn Path Menus. In Tabletop 2007, 121-128.

26. Matsushita, M., et al., Lumisight table: a face-to-face collaboration support system that optimizes direction of projected information to each stakeholder. In $C S C W$ 2004, 274-283.

27. Mazalek, A., M. Reynolds, \& G. Davenport, The TViews Table in the Home. In Tabletop 2007, 52-59.

28. Microsoft Surface. Available from: http://www.microsoft.com/surface (August 2008).

29. Morris, M.R., A.J.B. Brush, \& B.R. Meyers, Reading Revisited: Evaluating the Usability of Digital Display Surfaces for Active Reading Tasks. Tabletop 2007, 79-86

30. Nacenta, M.A., et al., A comparison of techniques for multi-display reaching. In $\mathrm{CHI} 2005,371-380$.

31. Nacenta, M.A., et al., The effects of interaction technique on coordination in tabletop groupware. GI 2007, 191-198

32. Olsen Jr, D.R., J. Clement, \& A. Pace, Spilling: Expanding Hand held Interaction to Touch Table Displays. In Tabletop 2007, 163-170.

33. Olwal, A. \& A.D. Wilson, SurfaceFusion: unobtrusive tracking of everyday objects in tangible user interfaces. In GI 2008, 235-242.

34. Park, D.G., et al., TAP: touch-and-play. In CHI 2006, 677-680.

35. Patten, J. \& H. Ishii, Mechanical constraints as computational constraints in tabletop tangible interfaces. In $\mathrm{CHI} 2007,809-818$.
36. Perry, M.J., Distributed Cognition and Computer Supported Collaborative Design: The Organisation of Work in Construction Engineering. PhD dissertation, 1997, Brunel University, UK.

37. Piper, A.M., et al., SIDES: a cooperative tabletop computer game for social skills development. In $\mathrm{CSCW}$ 2006, 1-10.

38. Ryall, K., et al., Exploring the effects of group size and table size on interactions with tabletop shared-display groupware. In CSCW 2004, 284-293.

39. Scott, S.D., M.S.T. Carpendale, \& K.M. Inkpen, Territoriality in collaborative tabletop workspaces. In CSCW 2004, 294-303.

40. Scott, S.D., S. Carpendale, \& S. Habelski, Storage Bins: Mobile Storage for Collaborative Tabletop Displays. IEEE Computer Graphics \& Applications, 2005, 25(4), 58-65.

41. Scott, S.D., K.D. Grant, \& R.L. Mandryk, System Guidelines for Co-located, Collaborative Work on a Tabletop Display. In ECSCW 2003, 159-178.

42. Sharp, H., Y. Rogers, \& J. Preece, Interaction design: Beyond human-computer interaction. 2nd ed. 2007, NY: Wiley.

43. Shen, C., K. Everitt, \& K. Ryall, UbiTable: Impromptu Face-to-Face Collaboration on Horizontal Interactive Surfaces. In UbiComp 2003.

44. Shen, C., et al., Informing the Design of Direct-Touch Tabletops. IEEE Computer Graphics \& Applications, 2006, 26(5), 36-46.

45. Sommer, R., Personal space: The behavior basis of design. 1969, Cliff, NJ: Prentice-Hall.

46. Tang, A., et al., Collaborative coupling over tabletop displays. In CHI 2006, 1181-1190.

47. Tang, J.C., Findings from Observational Studies of Collaborative Work. International Journal of ManMachine Studies, 1991, 34, 143-160.

48. Tse, E. \& G. Morrison, Using Public and Private Displays in a Collaborative Learning Environment. In PPD Workshop 2008, 1-3.

49. Tse, E., et al., Multimodal Split View Tabletop Interaction Over Existing Applications. In Tabletop 2007, 129-136.

50. Tse, E., et al., Enabling interaction with single user applications through speech and gestures on a multi-user tabletop. In AVI 2006, 336-343.

51. Wigdor, D., et al., Living with a Tabletop: Analysis and Observations of Long Term Office Use of a Multi-Touch Table. In Tabletop 2007, 60-67.

52. Wigdor, D., et al., Table-centric interactive spaces for real-time collaboration. In AVI 2006, 103-107.

53. Wilson, S., J. Galliers, \& J. Fone, Not all sharing is equal: the impact of a large display on small group collaborative work. In CSCW 2006, 25-28.

54. Yates, J.F., R.L. Klatzky, and C.A. Young, Cognitive Performance Under Stress. In R.S. Nickerson, (ed.) Emerging Needs and Opportunities for Human Factors Research, 1995, National Academy Press, 262-290.

55. Zajonc, R., Social facilitation. Science, 1965, 149, 269275. 